\title{
Sexualidade do público LGBTQIA+ e a consulta multiprofissional: (des)evolução?
}

Sexuality of the LGBTQIA+ public and the multiprofessional consultation: (de)evolution?

Sexualidad del público LGBTQIA + y la consulta multiprofesional: ¿(des) evolución?

Geovanna Renaissa Ferreira Caldas

ORCID: https://orcid.org/0000-0001-9820-309X

Centro de Ensino Tecnológico, Brasil

E-mail: geovannacaldas@ hotmail.com

Ananda Caroline Vasques Dantas Coelho

ORCID: https://orcid.org/0000-0003-3874-2299

Universidade Estadual do Ceará, Brasil

E-mail: nandakaroline2@gmail.com

Iranildo Lopes de Oliveira

ORCID: https://orcid.org/0000-0002-5106-2653

Secretaria Municipal de Saúde de Cascavel, Brasil E-mail: iranildooliver@hotmail.com

Maria Laura do Amparo Delfino

ORCID: https://orcid.org/0000-0002-7699-8829

Pontifícia Universidade Católica de Minas Gerais, Brasil

E-mail: ml.amparodelfino@gmail.com

Aniedne Hellen Cavalcanti

ORCID: https://orcid.org/0000-0003-3657-1625 Centro Universitário Brasileiro, Brasil

E-mail: aniedneh@gmail.com

Solange de Fátima Gomes

ORCID: https://orcid.org/0000-0002-0493-835X

Universidade Salgado de Oliveira, Brasil

E-mail: solange_enfermeira2008@hotmail.com

Luana Cristine Gomes da Silva

ORCID: https://orcid.org/0000-0002-0380-4819 Universidade Tiradentes, Brasil

E-mail: luanacristine_13@live.com

Cintia de Souza Melo

ORCID: https://orcid.org/0000-0003-3715-9851 Centro Universitário Brasileiro, Brasil E-mail: cintia_melo29@hotmail.com

Letícia Gabrielle César de Carvalho Menezes

ORCID: https://orcid.org/0000-0001-7287-950X

Universidade Católica de Pernambuco, Brasil E-mail: leticiacesar1804@gmail.com

Ana Bessa Muniz

ORCID: https://orcid.org/0000-0003-4414-9854 Instituto de Ciências e Tecnologia de São José dos Campos, Brasil Universidade Estadual Paulista, Brasil

E-mail: bessa.muniz@unesp.br

Andrio Corrêa Barros

ORCID: https://orcid.org/0000-0002-2063-3177

Faculdade Pitágoras, Brasil

E-mail: andriobarros@hotmail.com

Ernildo Weba Ferreira Junior

ORCID: https://orcid.org/0000-0002-5340-5138

Faculdade Florence, Brasil

E-mail: juniorweba@hotmail.com

Luana Rafaella Sampaio Leal

ORCID: https://orcid.org/0000-0002-8223-8471

Faculdade Estácio de Sá São Luís, Brasil

E-mail: lealluana71@gmail.com

\section{Resumo}

Objetivou-se refletir sobre as abordagens aplicadas dentro da consulta multiprofissionais frente a sexualidade da população LGBTQIA+. Trata-se de uma revisão integrativa de literatura, através de busca dentro das bases de dados: Banco de Dados em Enfermagem, Literatura Latino-Americana do Caribe em Ciências da Saúde e Medical Literature Analysis and Retrieval System Online. Dentre os critérios de inclusão: abordassem a temática central do estudo, 
publicados entre 2016 a 2021 e em português, inglês e/ou espanhol. Obtendo-se a totalidade de 7 documentos. O acesso da população se dá, principalmente, através das Unidades Básicas de Saúde (UBS) pela Estratégia de Saúde da Família. Dentro dessas unidades, os profissionais de enfermagem, medicina, fisioterapia, nutrição, odontológia, serviço social, entre outros, desempenham diversas funções, dentre elas a sua consulta, ou seja, eles estarão em contato direto com o público LGBTQIA+. Conclui-se que o mundo vem evoluindo em todos os aspectos, entretanto, ainda existe um forte estigma social que adentra a vida das pessoas e acaba gerando um preconceito, sendo esse, velado ou não. Essa discriminação também está presente dentro das unidades de saúde e das consultas prestadas pela equipe multidisciplinar de saúde.

Palavras-chave: Consulta multiprofissional; Público LGBTQIA+; Saúde; LGBT.

\begin{abstract}
The objective was to reflect on the approaches applied within the multidisciplinary consultation facing the sexuality of the LGBTQIA+ population. This is an integrative literature review, through a search within the databases: Database in Nursing, Latin American Caribbean Literature in Health Sciences and Medical Literature Analysis and Retrieval System Online. Among the inclusion criteria: addressed the central theme of the study, published between 2016 to 2021 and in Portuguese, English and/or Spanish. Obtaining the totality of 7 documents. The population's access is mainly through the Basic Health Units (UBS) through the Family Health Strategy. Within these units, professionals in nursing, medicine, physiotherapy, nutrition, dentistry, social work, among others, perform various functions, including your consultation, that is, they will be in direct contact with the LGBTQIA+ public. It is concluded that the world has been evolving in all aspects, however, there is still a strong social stigma that enters people's lives and ends up generating prejudice, whether this is veiled or not. This discrimination is also present within health units and consultations provided by the multidisciplinary health team.
\end{abstract}

Keywords: Multiprofessional consultation; LGBTQIA+ audience; Health; LGBT.

\title{
Resumen
}

El objetivo fue reflexionar sobre los enfoques aplicados dentro de la consulta multidisciplinaria frente a la sexualidad de la población LGBTQIA +. Se trata de una revisión integradora de la literatura, a través de una búsqueda dentro de las bases de datos: Base de Datos en Enfermería, Literatura del Caribe Latinoamericano en Ciencias de la Salud y Sistema de Análisis y Recuperación de Literatura Médica en Línea. Entre los criterios de inclusión: abordó el tema central del estudio, publicado entre 2016 a 2021 y en portugués, inglés y / o español. Obtención de la totalidad de 7 documentos. El acceso de la población es principalmente a través de las Unidades Básicas de Salud (UBS) a través de la Estrategia Salud de la Familia. Dentro de estas unidades, profesionales de enfermería, medicina, fisioterapia, nutrición, odontología, trabajo social, entre otros, realizan diversas funciones, incluida su consulta, es decir, estarán en contacto directo con el público LGBTQIA +. Se concluye que el mundo ha ido evolucionando en todos los aspectos, sin embargo, aún existe un fuerte estigma social que entra en la vida de las personas y termina generando prejuicios, sea este velado o no. Esta discriminación también está presente en las unidades de salud y las consultas que brinda el equipo multidisciplinario de salud.

Palabras clave: Consulta multiprofesional; Audiencia LGBTQIA +; Salud; LGBT.

\section{Introdução}

O termo "sexo" possui mais de um significado, visto que, ele pode estar relacionado ao gênero no sentido da biologia (feminino ou masculino), entretanto também está associada a atividade sexual. Adentrando a terminologia "sexualidade", ela é mais ampla, pois envolve o próprio sexo biológico, as identidades de gênero, as orientações sexual, entre outros (Pereira, 2019).

Segundo Souza e Meglhioratti (2017), o sexo biológico está relacionado as características alusivas desenvolvidas desde o momento da gestação e confirmadas por intermeio de ultrassonografias e após o seu nascimento, podendo então ser considerado como mulher, homem ou intersexual (os dois anteriores concomitantemente) e abrangem os cromossomos X e/ou Y, a genitália (pênis ou vagina), os hormônios e seus quantitativos e diversos outros fatores.

Por muitos anos o termo "gênero" era utilizado erroneamente, sendo apresentado como um sinônimo da palavra sexo, todavia, o sexo relaciona-se ao sentido da biologia, já a palavra gênero relaciona-se as áreas da psicologia e da sociologia, mas compreendem ainda o desempenho representado pelo que conhecemos como o feminino e masculino, o "papel da mulher" e “papel do homem” dentro da cultura da sociedade (Gonçalves \& Gonçalves, 2021).

Com o avançar do tempo, também aconteceu uma evolução do entendimento sobre o assunto, compreendeu-se a necessidade e importância de não restringir a apenas essas duas opções, modificando então, para Cisgênero (pessoa que 
identifica-se com o sexo de nascença), Transgênero (pessoa que identifica-se com um sexo diferente do de nascença), NãoBinário (pessoa que não identifica-se com nenhum dos dois ou vive com uma mistura dos dois), entre outros (Gonçalves \& Gonçalves, 2021).

Por fim, a orientação sexual remete ao socioafetivo e a atração emocional e/ou sexual por outro alguém. Anteriormente era conhecido popularmente por opção sexual, porém, entendeu-se que não se trata de algo a ser escolhido, não é uma opção (Melo \& Sobreira, 2018).

A orientação varia de pessoa para pessoa e possui diversos preponderantes, como por exemplo: Heterossexualidade (pessoa que se atrai fisicamente e emocionalmente por outros do sexo/gênero oposto), Homossexualidade (pessoa que se atrai fisicamente e emocionalmente por 5 outros do mesmo sexo/gênero), Bissexualidade (pessoa que se atrai fisicamente e emocionalmente por pessoas dos dois sexos/gêneros), Pansexualidade (pessoa que se atrai fisicamente e emocionalmente por pessoas, sem dar importância para gêneros/sexos), Assexual (pessoa que não se sente atraída fisicamente e emocionalmente por nenhum dos genêros/sexos), Demisexual (pessoa que só consegue se relacionar sexualmente com alguém que tenha conexão), e diversos outros (Reis, 2018).

A evolução sobre os entendimentos da temática levaram ao surgimento dos nomes "gays" e "lésbicas", que foram modificando até chegar ao acrônimo LGBTQIA+, incluindo Lésbicas, Gays, Bissexuais, Bigêneros, Transexuais, Travestis, Transgêneros, Queer, Intersexo e Assexuados (Schulman, 2013).

Compreende-se então, que a sociedade vive em constante atualização sobre tal assunto e consequentemente os outros segmentos da vida também devem ser constantemente atualizados, para o acompanhamento desse processo. Um desses segmentos é a consulta de enfermagem sobre sexualidade para e com esse público, respeitando as diferenças e abandonando os mitos, conhecimentos ultrapassados, preconceitos e tabus (Garcia \& Lisboa, 2012).

Segundo Silva et al (2021), a problemática enfrentada por esse público diz respeito justamente a discriminação e o preconceito vivenciado dentro de instituições que desempenham serviços de saúde. Pondera-se que a melhoria da inclusão do público nas consultas multiprofissionais, sofre influência direta do acolhimento, cuidado e forma de pensar dos profissionais da área da saúde.

Esse tipo de discriminação acontece devido ao conjunto de ideias ultrapassadas e preconceituosas que foram impregnadas na sociedade em anos anteriores, entretanto, também acontece devido à falta de conhecimento e orientações dos profissionais, onde os mesmos não possuem preparo para lidar com o público, faltando estímulo e capacitação durante a graduação e tomada de frente do próprio enfermeiro para buscar atuações e aperfeiçoamentos (Assis; et al, 2017).

Todo esse contexto gera um desconforto generalizado, principalmente voltados para o sentimental do usuário que está sendo atendido, o mesmo sente-se abandonado pelo serviço que deveria acolher a todos igualmente, têm os seus direitos feridos e desenvolve uma angústia e receio das instituições de saúde, o que afeta diretamente a sua própria saúde (Vitiritti, Andrade \& Peres, 2016).

Sendo assim, compreende-se a necessidade de pesquisas voltadas para esse público que sempre foram abandonados pela sociedade em todos os aspectos, áreas e sentidos da vida. Visto que, dentre da área da saúde, isso se repete, deixando de lado os princípios do Sistema Único de Saúde e gerando carências que poderiam ser resolvidas. Entendendo a relevância da temática abordada, surgiu a seguinte questão norteadora: "Como se dá a consulta de enfermagem sobre sexualidade voltada especificamente para o população LGBTQIA+?".

Objetivou-se refletir sobre as abordagens aplicadas dentro da consulta multiprofissionais frente a sexualidade da população LGBTQIA+. 


\section{Metodologia}

O presente estudo trata-se de uma revisão integrativa de literatura, sendo essa, um tipo de revisão de abordagem metodológica que possibilita a explanação da temática, através da elucidação dos conceitos, pesquisa por dados e documentos da literatura, utilizando estudos experimentais e não-experimentais, analisando os mesmos e apresentando resultados sobre o conteúdo estudado (Souza et al., 2010).

Para obtenção dos resultados desejados, realizou-se uma busca dentro das bases de dados: Banco de Dados em Enfermagem (BDENF), Literatura Latino-Americana do Caribe em Ciências da Saúde (LILACS) e Medical Literature Analysis and Retrieval System Online (MEDLINE), todos via Biblioteca Virtual em Saúde (BVS), sendo essa, uma biblioteca que reúne diversas bases de dados na área da saúde.

Essa busca ocorreu por intermédio do uso dos Descritores em Ciências da Saúde (DeCS): "Consulta", "Pessoas LGBTQIA+ e Saúde, em associação a utilização do operador booleano "AND”, apresentando-se da seguinte forma e sequência "Consulta AND Pessoas LGBTQIA+ AND Saúde".

Os critérios de inclusão utilizados para seleção e inclusão dos estudos foram: Artigos que abordassem a temática central do estudo e que respondessem a questão norteadora e estivessem relacionado com o objetivo, publicados entre os anos de 2016 a 2021 e com as linguagens de português, inglês e/ou espanhol.

Já dentre os critérios de exclusão, inclui-se: Estudos repetidos, outras revisões de literatura, resumos simples ou expandidos publicados em anais de eventos em revistas, documentos incompletos, estudos inconclusivos e artigos que não tivessem relação com a temática previamente selecionada.

Após busca nas bases de dados com aplicação dos descritores e operador booleano, realizou-se a leitura dos títulos e resumos dos estudos, levando em consideração os critérios de inclusão e exclusão, para a seleção dos documentos que irão compor o estudo.

\section{Resultados}

Após todo o processo de seleção, obteve-se uma totalidade de 7 documentos para compor o estudo, sendo destes, 3 na língua portuguesa e 4 na língua inglesa, 2 na base de dados LILACS, 4 na base de dados MEDLINE e 1 na base de dados BDENF.

Esses foram organizados e apresentados no Quadro 1, proporcionando um panorama geral das publicações selecionadas, destacando os fatores de autores e ano de publicação, periódico que o mesmo se encontra, linguagem principal, base de dados de onde foi retirado e objetivo dos estudos. 
Quadro 1: Caracterização dos estudos utilizados.

\begin{tabular}{|c|c|c|c|c|c|}
\hline $\mathbf{N}^{\circ}$ & Autores e Ano & Periódico & Linguagem & Base de dados & $\begin{array}{l}\text { Objetivo } \\
\end{array}$ \\
\hline 1 & Cabral; et al., 2019. & Rev. enferm. UFPE on line & Português & BDENF & $\begin{array}{l}\text { Analisar, sob a ótica de mulheres lésbicas e } \\
\text { bissexuais, a assistência de Enfermagem em } \\
\text { Unidades de Saúde da Família. }\end{array}$ \\
\hline 2 & $\begin{array}{l}\text { Nascimento; et al., } \\
2020 .\end{array}$ & Rev. enferm. UFSM & Português & LILACS & $\begin{array}{l}\text { Descrever as percepções das enfermeiras residentes } \\
\text { em processo de qualificação para a assistência pré- } \\
\text { natal acerca das questões de gênero na consulta de } \\
\text { enfermagem. }\end{array}$ \\
\hline 3 & $\begin{array}{l}\text { Palacios-Baena, } \\
\text { Martín-Ortega \& } \\
\text { Ríos-Villegas, } \\
2020 .\end{array}$ & Med Clin (Barc) & Inglês & MEDLINE & $\begin{array}{l}\text { Definir as características clínicas de novos } \\
\text { diagnósticos e esclarecer os fatores de risco (FR) } \\
\text { associados à DL com ou sem doença avançada (DA) } \\
\text { entre } 2014 \text { e } 2018 \text {. }\end{array}$ \\
\hline 4 & John; et al., 2020. & Sex Transm Infect & Inglês & MEDLINE & $\begin{array}{l}\text { Determinar a disposição de homens gays e bissexuais } \\
\text { (GBM) para fornecer kits de autoteste de HIV } \\
\text { (HIVST) com terapia de parceiro entregue pelo } \\
\text { paciente (PDPT) e se envolver em notificação de } \\
\text { parceiro baseada em aplicativo de rede sexual } \\
\text { geossocial (GSN). }\end{array}$ \\
\hline 5 & $\begin{array}{l}\text { Taverne; et al., } \\
2021 .\end{array}$ & BMJ Open & Inglês & MEDLINE & $\begin{array}{l}\text { Avaliar o impacto do nacional de saúde sistema de } \\
\text { cobertura em saúde despesas de pessoas vivendo com } \\
\text { HIV, medindo a quantidade despesas do próprio } \\
\text { bolso para uma rotina de consultas em Dakar e } \\
\text { diferentes regiões do Senegal. }\end{array}$ \\
\hline 6 & Agustí; et al., 2021. & $\begin{array}{l}\text { Enferm Infecc Microbiol } \\
\text { Clin (Engl Ed) }\end{array}$ & Inglês & MEDLINE & $\begin{array}{l}\text { Descrever a viabilidade e a relação custo - eficácia da } \\
\text { oferta de testes de HIV em intervenções de extensão } \\
\text { e subsequente consulta dos resultados por meio de } \\
\text { uma página da web segura. }\end{array}$ \\
\hline 7 & Silva; et al., 2021. & REVISA (Online) & Português & LILACS & $\begin{array}{l}\text { Descrever a produção do cuidado em Enfermagem à } \\
\text { saúde de Lésbicas, Gays, Bissexuais, Travestis e } \\
\text { Pessoas Trans Queers Intersexos, Assexuais e outras } \\
\text { identidades sexuais e de gênero, a partir das reflexões } \\
\text { acerca do trabalho da enfermeira. }\end{array}$ \\
\hline
\end{tabular}

Fonte: Autores.

\section{Discussão}

O acesso da população se dá, principalmente, através das Unidades Básicas de Saúde (UBS) pela Estratégia de Saúde da Família (ESF). Dentro dessas unidades, os profissionais de enfermagem, medicina, fisioterapia, nutrição, odontológia, serviço social, entre outros, desempenham diversas funções, dentre elas a sua consulta, ou seja, eles estarão em contato direto com o público LGBTQIA+ (Silva et al., 2018).

Em relação a consulta de enfermagem, Cabral et al (2019) descrevem a opinião de mulheres lésbicas e bissexuais, as mesmas relataram uma ausência no quesito de acolhimento, preconceito escancarado, sendo necessário esconder a orientação sexual e principalmente, falta de preparo profissional, pois os profissionais tendem a considerar que aquela pessoa seja hetero cis, não perguntando e consequentemente, repassando orientações que não fazem parte da realidade daqueles pacientes. A pesquisa também apresenta dados de que, em relação a realização de testes rápidos e consulta citopatológica, elas tendem a não voltar para buscar o resultado, justamente pelo preconceito sofrido durante a primeira consulta.

Entende-se que a temática é extremamente complexa, inclusive voltando o olhar para o momento de realização de testes rápidos para e com esse público, a pesquisa de Agustí et al (2021), evidencia a alternativa da realização de testes em laboratório de referência para homens gays e mulheres trans, com obtenção de resultados via online.

Essa opção configura-se como uma alternativa positiva, devido ao estigma que existiu durante muito tempo sobre essas pessoas desempenharem comportamentos questionáveis e isso aumentar o risco da transmissão de doenças sexualmente transmissíveis.

Confirmando essa mesma abordagem, John et al (2020) também dissertam sobre os pontos positivos de considerar novas estratégias para a realização e divulgação do resultado de testes rápidos para esse público, esses são viáveis devido ao custo, principalmente relacionado a efetividade, mas também iria gerar um maior número de pessoas que chegam até a etapa de pegar 
o resultado e buscar tratamento adequado, visto que, segundo Taverne et al (2021) a cobertura ainda é baixa e possui pouca eficácia e limitado. Isso ocorre frequentemente, pois os mesmos entendem que dessa forma, não iriam passar pela vergonha e pelo risco de sofrer qualquer tipo de prejulgamento pela sua orientação sexual (Palacios-Baena et al., 2020).

Esse pensamento vem caindo conforme a evolução humana, entretanto, muitos profissionais ainda permanecem com ideias retrógradas e preconceituosas, afetando o andamento da consulta e a realização desses testes voltados para as infecções sexualmente transmissíveis (IST’s) (Guimarães, 2018).

Adentrando a consulta médica, existe uma grande fragilidade durante a prestação desse cuidado. Isso pode ocorrer devido a falta de informação dentro da graduação, Negreiros et al (2019) abordam falas dos próprios médicos relatando que a temática nunca foi abordada dentro de sala de aula ou que nunca sequer ouviram a sigla LGBT e que mesmo quando era abordado, tudo era muito rápido e sucinto. Tal afirmativa apenas evidencia a carência que existe dentro das universidades, demonstrando que esse público continua sendo esquecido até mesmo durante os estudos e que os profissionais da saúde saem despreparados para lidar com a situação.

Nascimento et al (2020) expõem a opinião de residentes na área da saúde, durante essas consultas na prestação da assistência e a importância de atuar frente os estereótipos de gênero, ainda sobre a mesma visão antiga de mulher ter que cumprir o papel da mulher e homem cumprir o papel do homem. A sociedade sempre impôs que a mulher fosse frágil, então muitos não sabem como abordar essa temática e muito menos se envolver com os quesitos de orientação, sexualidade e gênero, de uma forma que saiba atuar de maneira humanizada e que saiba orientar aquela pessoa, com equidade, baseado na sua realidade e necessidade.

Percebe-se que as principais problemáticas relacionadas a esse assunto, envolvem: Estereótipos, as pessoas acabam sendo julgadas pelo seu jeito de agir; Invisibilidade da identidade, a pesquisa realizada por Silva et al (2021) apresenta falas de enfermeiras relatando que a orientação sexual não é algo digno de ser investigado no histórico, ou seja, eles são invisibilizados em todos os aspectos; Desconhecimento das demandas de atendimento direcionado aos LGBTQIA+, profissionais demonstram ficar assustados ao prestarem consulta com o público, que acham invasivo, então preferem não perguntar ou comentar, isso faz com que a consulta não seja voltado para tais necessidades.

Silva et al (2021) não discorrem apenas de dados negativos, os autores também citam opções válidas para a busca na melhoria desses obstáculos e complicações. Dentre eles, cita-se: Acolhimento digno e humanizado; Práticas de assistência em saúde sexual e reprodutiva, com realização de testes rápidos, orientações e distribuição de preservativos; Assistência realizada a hormonização, sobretudo para os transsexuais; Reconhecimento de vulnerabilidade em saúde, relacionados às necessidades da vivência, financeira e de risco de vida e de integridade física e sexual, devido ao preconceito, que proporciona diversos casos de violência.

\section{Considerações Finais}

Conclui-se que o mundo vem evoluindo em todos os aspectos, entretanto, ainda existe um forte estigma social que adentra a vida das pessoas e acaba gerando um preconceito, sendo esse, velado ou não. Essa discriminação também está presente dentro das unidades de saúde e das consultas prestadas pela equipe multidisciplinar de saúde.

Compreende-se que há uma extrema necessidade de conversas e orientações ainda enquanto estudantes, dentro das universidades, pois é possível visualizar uma grande carência frente ao saber atuar e conversar com o público LGBTQIA+, de forma com que a assistência seja realizada de maneira completa, digna, humanizada, respeitando o outro e atuando através do princípio da equidade.

Sobre a temática, percebe-se uma limitação nos estudos, pois evidenciou-se uma pequena quantidade de estudos que abordam o assunto proposto, então, carece de mais estudos sobre o mesmo. O público LGBTQIA+ já vem sendo desmerecido e 
apagado em todos os setores e áreas da sua vida, a falta de pesquisas sobre eles, só confirma mais uma invisibilidade e falta de estímulo sobre.

Como sugestões para trabalhos futuros, recomenda-se a realização de mais pesquisas sobre o público, principalmente sobre essa diferenciação, através de um estudo comparativo, entre a consulta para o público heterosexual e o público homossexual, bisexual, transsexual...

\section{Referências}

Assis, B. F., et al. (2017). Homossexualidade feminina e a consulta ginecológica: uma revisão integrativa. International Nursing Congress, 9 (12), may.

Agustí, C., et al. (2021). Outreach HIV testing using oral fluid and online consultation of the results: Pilot intervention in Catalonia. Enferm Infecc Microbiol Clin (Engl Ed), 39 (1): 3-8.

Cabral, K. T. F., et al. (2019). Assistência de enfermagem às mulheres lésbicas e bissexuais. Rev. Enferm. UFPE on line, 13 (1): $79-85$.

Garcia, O. R. Z. \& Lisboa, L. C. S. (2012). Consulta de Enfermagem em sexualidade: Um instrumento para assistência de Enfermagem à Saúde da Mulher, em nível de Atenção Primária. Texto \& Contexto - Enfermagem, 21 (3): 708-716.

Gonçalves, M. C. \& Gonçalves, J. P. (2021). Gênero, Identidade de Gênero e Sexualidade: Conceitos e determinações em contexto social. Revista Ciências Humanas - Educação e Desenvolvimento Humano, 14 (25).

Guimarães, R. C. P. G. (2018). Estigma e Diversidade Sexual nos Discursos dos (as) profissionais do SUS: Desafios para a saúde da população LGBT. Tese apresentada para Doutorado em Saúde Coletiva pela Universidade de Brasília, Brasília, 07 nov.

John, S. A., et al. (2020). High willingness to use novel HIV and bacterial sexually transmitted infection partner notification, testing, and treatment strategies among gay and bisexual men. Sex Transm Infect., 96 (3): 173-176.

Melo, T. G. R. \& Sobreira, M. V. S. (2018). Identidade de Gênero e Orientação Sexual: Perspectivas literárias. Temas em Saúde, 18(3).

Nascimento, C. M., et al. (2020). Questões de gênero na consulta pré-natal de enfermagem: percepções das enfermeiras residentes. Rev. enferm. UFSM, 10: e91.

Negreiros, F. R. N., et al. (2019). Saúde de Lésbicas, Gays, Bissexuais, Travestis e Transexuais: da Formação Médica à Atuação Profissional. Revista Brasileira de Educação Médica, 43 (1): 23-31.

Palacios-Baena, Z. R., Martín-Ortega, M. \& Ríos-Villegas, M. J. (2020). Profile of new HIV diagnoses and risk factors associated with late diagnosis in a specialized outpatient clinic during the 2014-2018 period. Med Clin (Barc), 155 (11): 482-487.

Pereira, D. (2019) Sexualidade e Relação de Gênero. Atena Editora, Ponta Grossa, 1.

Reis, T. (2018). Manual de Comunicação LGBTI+. Aliança Nacional LGBTI / GayLatino.

Schulman, M. (2013). Assexuados, bichas \& cia: a nova geração gay nasuniversidades dos EUA. Tradução: Clara Alain. Texto originalmente publicado no jornal "The New York Times". Folha de S. Paulo. Seção Comportamento.

Silva, A. A. C., et al. (2021). Produção do cuidado de enfermagem à população LGBTQIA+ na atenção primária. REVISA (Online), 10 (2): $291-303$.

Silva, D. D., et al. (2018). Assistência de Enfermagem na Atenção Básica a populaçã homossexual da cidade de Caicó-RN. Temas em Saúde, 18 (3).

Souza, M. T., Silva, M. D., \& Carvalho, R. (2010). Revisão integrativa: o que é e como fazer. Einstein, 8(1): 102-6.

Souza, B. B., \& Meglhioratti, F. A. (2017). Uma reflexão a respeito dos conceitos de Sexo Biológico, Identidade de gênero e Identidade afetivo-sexual. Simpósio Internacional e Educação Sexual - saberes/trans/versáteis currículos identitários e pluralidades de gênero, abr.

Taverne, B., et al. (2021). Does universal health coverage reduce out-of-pocket expenditures for medical consultations for people living with HIV in Senegal? An exploratory cross-sectional study. BMJ Open, 11 (7): e046579.

Vitiritti, B., Andrade, S. M. O., \& Peres, J. E. C. (2016). Diversidade sexual e relações profissionais: Concepções de médicos e enfermeiros. Temas psicol., 24 (4): 1389-1405. 